Para enlazar con este artículo / To link to this article:

http://dx.doi.org/10.6035/MonTI.2019.11.10

Para citar este artículo / To cite this article:

Pérez Fernández, Lucila María. (2019) "El vídeo análisis: Un nuevo enfoque para la didáctica de la interpretación." In: Tolosa Igualada, Miguel \& Álvaro Echeverri (eds.) 2019. Porque algo tiene que cambiar. La formación de traductores e intérpretes: Presente \& futuro / Because something should change: Present \& Future Training of Translators and Interpreters. MonTI 11, pp. 263-279.

\title{
EL VÍDEO ANÁLISIS: UN NUEVO ENFOQUE PARA LA DIDÁCTICA DE LA INTERPRETACIÓN CONSECUTIVA
}

\author{
Lucila María Pérez Fernández \\ Lucila.perez@uneatlantico.es \\ Universidad Europea del Atlántico
}

\section{Resumen}

El sistema de créditos ECTS unido al rápido desarrollo de las nuevas tecnologías favorecen un cambio de metodologías docentes. En ellas se promueve el trabajo autónomo, pero en el caso de la asignatura de Interpretación Consecutiva, los alumnos demandan actividades más guiadas que les permitan desarrollar las habilidades necesarias para su labor de intérpretes. En los últimos años, el vídeo análisis ha ido perfilándose como una valiosa herramienta en el ámbito educativo. Por eso, se ha diseñado una propuesta didáctica en la que se combina este recurso con un diario de aprendizaje que permita desarrollar la metacognición del alumnado, con el objetivo de mejorar sus capacidades de observación, análisis y reflexión y, en consecuencia, controlar su propio aprendizaje.

\begin{abstract}
"Video analysis: a new approach towards consecutive interpreting didactics"

The ECTS credit system together with the rapid development of new technologies favor a change in teaching methodologies allowing students greater autonomy and responsibility. In a Consecutive Interpretation course, students demand more guided activities that allow them to develop the necessary skills for their work as interpreters. In recent years, video analysis has emerged as a valuable tool in the field of education. For this reason, a didactic proposal has been designed in which this resource is combined with a learning diary that allows students to develop metacognition, with the aim of improving their observation, analysis and reflection skills and, consequently, controlling their own learning.
\end{abstract}


Palabras clave: Interpretación. Vídeo análisis. Diario de aprendizaje. Metacognición. Codimg.

Keywords: Interpretation. Video analysis. Learning diary. Metacognition. Codimg. 


\section{Introducción}

El peso que tienen las asignaturas de Interpretación en los planes de estudios de los grados de Traducción e Interpretación es bastante inferior al de las asignaturas específicas de Traducción. Si tenemos en cuenta que las destrezas que nuestros alumnos deberán desarrollar en ambas ramas son diferentes, salta a la vista que el tiempo de clase es insuficiente y requiere complementarse con trabajo autónomo del alumno. Sin embargo, muchas veces los alumnos demandan actividades más guiadas que puedan realizar fuera del aula, pero en las que, a su vez, obtengan algún tipo de feedback. Esto ha hecho que, en la última década, sean muchos los docentes que hayan propuesto nuevos enfoques metodológicos basados en la autonomía que han de adquirir los estudiantes (Palomares 2011; Ramón 2010; Rodríguez 2006). Aunque ninguno de ellos versa sobre la metodología de la interpretación en particular, sí que nos sirven para observar la evolución progresiva en las metodologías aplicadas por los docentes.

Si nos centramos más en la materia que nos ocupa, observamos que en las investigaciones más recientes en didáctica de la interpretación son pocos los autores que profundizan en la introducción del componente reflexivo y el fomento de la metacognición en el aula. Sin embargo, tal y como indica Arumi (2009), "la metacognición, o el conocimiento del conocimiento (Flavell 2000), es una estrategia eficaz que permite al intérprete controlar su propia cognición con el objetivo de mejorar su rendimiento". Por lo tanto, se trata de un aspecto al que los docentes deberíamos prestar más atención.

En este sentido, dada la necesidad de fomentar el trabajo autónomo de los alumnos, así como la práctica reflexiva, presentamos una propuesta didáctica basada en el uso del video análisis como herramienta de autoevaluación y centrada en la metacognición mediante el uso del diario de aprendizaje como herramienta de reflexión, que permita al alumno controlar su propio aprendizaje, con el fin de mejorar su rendimiento. Nuestra intención es, mediante la combinación de ambos recursos, lograr los siguientes objetivos: 
- Desarrollar la capacidad de reflexión del alumnado, con el fin de favorecer la toma de decisiones a la hora de interpretar.

- Fomentar la autonomía del alumnado, a través de una enseñanza reflexiva.

\section{Evolución de las tendencias metodológicas en la didáctica de la interpretación}

Los intérpretes existen desde que hay fronteras. De acuerdo con Baigorri (2000: $1)$, es probable que su labor sea incluso más antigua que la del traductor. Kalina (2015) concluye entonces que existen pruebas de que la formación de intérpretes se remonta a tiempos muy lejanos. Ya en el siglo IX se formaba a alumnos en el aprendizaje de lenguas, que luego emplearían en su labor de intérpretes. Esta formación se centraba tanto en el dominio de la lengua extranjera como en el aprendizaje de habilidades específicas para la interpretación (Wiotte-Franz 2001).

Los esfuerzos para formar a intérpretes en instituciones académicas data del siglo XIII, cuando se estableció el denominado Oriental College, en el que los alumnos recibían formación en lenguas, derecho, ciencias, matemáticas, teología y medicina (Kalina 2015: 18).

También Cristobal Colón buscó formación para los nativos americanos que trajo de sus viajes, con la intención de que una vez que aprendiesen español, pudiesen ejercer como intérpretes.

Como indica Domínguez (2015: 64) se suele considerar la Conferencia de Paz de París como el hito fundacional de la profesión y de la preparación para esta, ya que a partir de ese momento la demanda de profesionales aumentó y, como señala Kalina (2015: 18), se hizo patente la necesidad de formación reglada. Como consecuencia se fundaron las primeras instituciones universitarias dedicadas a la enseñanza de la interpretación en Europa. Domínguez (2015: 64) enumera las siguientes: Mannheim en 1930; Ginebra en 1941; Gante en 1941 y Viena en 1943; Gemersheim en 1946; Graz en 1947 y Saarbrücken en 1948. También fuera de Europa se empezaron a ofrecer cursos de interpretación específicos.

En los años 50 del siglo XX se consolidan las primeras escuelas de interpretación: la ESIT (École Supérieure d'Interprètes et de Traducteurs) en 1958 y el ISIT (Institut Supérieur d'Interprétation et de Traduction) en 1959. En lo que respecta a la didáctica empleada en estas escuelas, Domínguez (2015: 65) señala que poco se sabe, no existía una pedagogía específica y la poca información de la que se tiene constancia apunta a que los profesores se centraban 
en enseñar prácticas que a ellos mismos les habían funcionado en su labor profesional.

En los años 70, surge la denominada Teoría del sentido (Seleskovitch 1975) vinculada a la Escuela de París, y muy inspirada en la práctica y según la cual, la interpretación no debe basarse exclusivamente en la parte lingüística, es decir, no se trata de traducir palabras, sino de reexpresar el contenido del discurso. Martin (2015: 91) indica que la influencia del modelo de Seleskovitch se percibe en varias prácticas docentes como la disociación de palabras y significado o la realización de ejercicios de preinterpretación.

Partiendo de este modelo, Lederer describió en los años 80 las ocho operaciones mentales que intervienen en el proceso de interpretación. Estas son: audición, comprensión de la lengua, conceptualización, enunciación a partir del recuerdo cognitivo, conciencia de la situación, control auditivo, transcodificación del mensaje a otro idioma y recuerdo de significados específicos (Lederer 1981: 50).

Para Arumí \& Domínguez (2013) es evidente que la didáctica de la interpretación se está academizando cada vez más, como así lo demuestra el número cada vez más elevado de tesis doctorales en las que se ahonda en la didáctica de la interpretación. En los últimos años ya es frecuente encontrar aportaciones de autores que recogen y sistematizan la práctica didáctica actual a partir de su trayectoria docente. Ejemplo de ello son Baxter (2012) y Alonso (1999, 2001, 2005, 2009).

\subsection{Uso de la tecnología en el aula de interpretación}

Domínguez (2015) señala la influencia de la evolución tecnológica en el diseño de los planes de estudio. Esta influencia no es algo reciente, sino que la tendencia de combinar la tecnología y la docencia de la interpretación data ya de los años 90 con el nacimiento de las herramientas CAIT (Computer Assisted Interpreter Training), cuyo objetivo, tal y como indica Sandrelli (2015: 116) es mejorar la docencia y servir de apoyo para el aprendizaje autónomo de los alumnos.

Las primeras herramientas CAIT consistían en repositorios de discursos. Un ejemplo es IRIS (Interpreter's Resource Information System), una base de datos multimedia interactiva, creada en 1999 por Angela Carabelli y F. Fioravanti en la Universidad de Trieste para la combinación de lenguas inglés-italiano (Moro y Torres del Rey 2008: 113). Antes de esa fecha, en 1995, De Ferra y Cervato, de la Universidad de Hull, desarrollaron el proyecto Interpr-it, enfocado, sobre todo, a la interpretación de enlace (aunque también se ofrecía un módulo de 
práctica de interpretación consecutiva) y que consistía en un CD con ocho entrevistas (italiano-inglés) y una serie de ejercicios para que los estudiantes pudieran practicar tanto dentro de clase como fuera. Tras la puesta en práctica de este programa, los autores concluyeron que su uso contribuía a la reducción de los niveles de ansiedad durante la interpretación (Sandrelli 2015: 122).

En 1999, en esa misma universidad, surgió el programa Interpretations de la mano de Sandrelly y Hawkins. Este se centraba en la interpretación simultánea y su principal diferencia con el anterior era que, mientras que Interpr-it se basaba en los soportes CD o DVD, con la nueva herramienta se pretendía ofrecer a los docentes una plataforma en la que pudiesen desarrollar sus propios materiales y estructurarlos en módulos y ejercicios (Sandrelli 2015: 122).

La acogida de esta herramienta fue muy buena, por lo que se decidió convertirla en un programa comercial: Black Box. Este incluía dos novedades principales, con respecto a su antecesor. Por un lado, la posibilidad de utilizarse como herramienta colaborativa (Interpretations se había concebido como un programa para uso individual exclusivamente) y por otro lado, la inclusión de un asistente de ejercicios (Exercise Wizard), con el fin de ayudar a los docentes a la hora de crear tareas.

En España, la Universidad de Granada creó en 2001 una base de datos llamada Marius, como herramienta de apoyo a las ya mencionadas Interpretations o Black Box. Así, como indica De Manuel (2006: 207) su principal propósito era acumular "textos individuales, procedentes todos de grabaciones en vídeo, analógicas o digitales", que después se clasificarían en función de diversos criterios (duración, número de palabras, velocidad de elocución, acento del orador, etc.), con el fin de "adjudicarlos a una modalidad de interpretación y fase del aprendizaje (desde consecutiva inicial hasta simultánea avanzada)".

También la Comisión Europea ofrece acceso gratuito a su Speech Repository 2.0, dirigido tanto a estudiantes de interpretación como a docentes, y en el que encontramos discursos de muy diferente índole y clasificados en función de las lenguas, tipo de interpretación, temática y nivel. Asimismo, la Comisión Europea cuenta con otro proyecto financiado por la Unión Europea denominado ORCIT (Recursos Online para la Formación de Intérpretes de Conferencias) cuyo objetivo es diseñar herramientas pedagógicas de ayuda tanto a estudiantes como a formadores.

Por otro lado, encontramos también aplicaciones para la toma de notas en interpretación consecutiva. Alguna, como Noteshelf, Penultimate o Evernote no son específicas para la interpretación, pero pueden ser de utilidad para la toma de notas en soporte tableta. No obstante, ya hay investigadores que se 
ha propuesto diseñar herramientas tecnológicas de aplicación en la interpretación. Un ejemplo lo encontramos en la aplicación Cleopatra diseñada por la intérprete Lourdes de la Torre, como un método de entrenamiento en automatización de símbolos.

Todos estos avances tecnológicos hacen que inevitablemente se produzca una evolución de las metodologías empleadas en las aulas, pues es lógico que los docentes se aprovechen de las múltiples ventajas que nos aporta la tecnología para el diseño de nuestras clases.

\section{Codimg, un nuevo software de video análisis}

Codimg es una herramienta de video análisis de reciente creación, que permite utilizarla en entornos académicos, con el fin de que tanto los estudiantes puedan mejorar su rendimiento gracias al análisis de diferentes parámetros a partir de grabaciones en vídeo. Por lo tanto, no solo es útil para profesores, ya que pueden ofrecer un feedback mucho más detallado, sino que también los alumnos se pueden beneficiar de este programa, pues pueden ver sus propios vídeos y autoevaluar su trabajo.

Una de las principales ventajas de esta herramienta es la facilidad con la que se puede utilizar. Simplemente se necesita grabar un vídeo, establecer los parámetros que queramos analizar e ir marcándolos a medida que los encontramos en el vídeo, de este modo, al finalizar podremos extraer los clips de vídeo que nos interesen para nuestro análisis.

Como indica Neal (2012: 65), las habilidades de un profesional de la interpretación consecutiva están muy relacionadas con las habilidades de comunicación interpersonal de los estudiantes, de ahí que la principal utilidad que nos aporta este programa es precisamente dar a los estudiantes la oportunidad de evaluar esas habilidades a las que muchas veces no prestan la misma atención que al contenido.

\subsection{Ventajas del vídeo análisis en el aula de interpretación consecutiva}

En los últimos años, el vídeo análisis ha ido perfilándose como una valiosa herramienta en el ámbito educativo. Por lo general, son los investigadores quienes, apoyándose en las imágenes y su interpretación, consiguen desvelar y documentar realidades imperceptibles (Baer y Schnettler 2014). No obstante, no se trata de un recurso cuyo único fin sea la investigación, sino que en también se puede emplear dentro del aula con fines didácticos.

A diferencia de la traducción, en la que podemos releer y analizar de nuevo nuestro texto meta todas las veces que queramos, la interpretación es volátil, es 
decir, cuanto más tiempo pasa menos recordaremos, lo que dificulta el proceso de reflexión y evaluación de los propios errores. En este sentido, el uso de una herramienta de vídeo análisis logra suplir esa carencia y nos permite analizar nuestro discurso, de tal modo que podamos ser conscientes de manías en las que no reparamos, no solo gestuales, sino también de otro tipo como, por ejemplo, el uso de muletillas o de otro tipo de errores que, por la velocidad y la concentración del momento, nos pasan desapercibidos (por ejemplo, errores de sentido o frases inacabadas).

Así pues, el vídeo análisis constituye una valiosa herramienta en el desarrollo de las habilidades de los futuros intérpretes, pues les exige poner en práctica sus capacidades de observación, análisis y reflexión y también contribuye a una mejor asimilación de los aprendizajes teóricos, en los cuales se tendrán que basar a la hora de autoevaluarse.

\section{Propuesta de integración de Codimg en las clases de interpretación consecutiva}

\subsection{Justificación}

El sistema de créditos ECTS promueve el trabajo autónomo del alumno. Sin embargo, en el caso de interpretación, a pesar de que los alumnos son conscientes de los tipos de actividades que pueden realizar fuera del aula (ejercicios para agilizar la memoria, prácticas de locución, ejercicios de voz y respiración, prácticas de interpretación en páginas especializadas, etc.), son pocos los que realmente llegan a llevarlos a cabo.

Si además tenemos en cuenta que las asignaturas de Interpretación escasean en los planes de estudio de los grados de Traducción e Interpretación, queda patente que, para que el alumnado logre adquirir las destrezas y competencias necesarias para ser intérprete, es imprescindible que los estudiantes desarrollen trabajo autónomo fuera de las clases. Por lo tanto, esta propuesta surge de la necesidad de proporcionar actividades de refuerzo que los alumnos puedan realizar en casa para mejorar su rendimiento en la asignatura de Interpretación Consecutiva.

Como docentes constatamos que muchas veces los alumnos se muestran muy preocupados por transmitir el contenido y dejan de lado los elementos formales de una interpretación consecutiva, tales como la postura o la dicción. Por lo tanto, mediante el uso de la herramienta que presentaremos a continuación, pretendemos ayudar a los alumnos a mejorar la calidad de sus interpretaciones. Cabe aquí mencionar que, por las características del 
programa, no abordaremos la parte de la toma de notas, por lo que esta destreza deberá trabajarse en el aula o fuera de ella a través de otros ejercicios específicos a propuesta del docente o mediante el uso de aplicaciones como la anteriormente mencionada Cleopatra.

\subsection{El proceso de interpretación consecutiva}

$\mathrm{Si}$ bien es cierto que existen numerosos estudios sobre el proceso de interpretación, constatamos que todavía no existe consenso sobre las fases que lo conforman. Por ejemplo, Seleskovitch y Lederer (1989) lo ven como un proceso compuesto de tres operaciones: la comprensión, la desverbalización y la reexpresión. Por su parte, Vanhecke y Lobato (2009) hablan de cuatro fases: escuchar, comprender, analizar y reexpresar. Gile (2009) hace referencia a tres esfuerzos: el de escucha y análisis, el esfuerzo de memoria y el esfuerzo de producción. Por último, Bosch (2012) divide los procesos cognitivos que se llevan a cabo en cualquier interpretación en tres grandes bloques: escucha, comprensión y reformulación. Sin embargo, señala que para la interpretación consecutiva estas fases varían y distingue una más: el análisis del discurso, que tiene lugar justo antes de la reformulación.

Para nuestra propuesta adoptaremos la división triangular de Bosch, si bien nos centraremos en la parte de reformulación, ya que consideramos que es este proceso el que se puede ver beneficiado del uso del video análisis como herramienta de aprendizaje.

\subsection{La autoevaluación como eje central del proceso de aprendizaje}

En el modelo educativo tradicional, los docentes asumimos el papel de transmisores del conocimiento y, de hecho, nuestras aportaciones y valoraciones del trabajo de los alumnos son útiles, aunque, como ya dijimos, en el caso de la asignatura que nos ocupa, insuficientes. Es aquí donde entra en juego la autoevaluación como recurso didáctico necesario para que los estudiantes de Traducción e Interpretación desarrollen las habilidades y destrezas necesarias para ejercer la labor de intérpretes.

Tal y como indican Falchikov \& Boud (1989: 529), entendemos por autoevaluación el proceso de implicación de los estudiantes a la hora de emitir juicios de valor sobre los logros y resultados de su aprendizaje. A pesar de que la habilidad de autoevaluarse no siempre se trabaja en las clases, lo cierto es que se trata de una de las destrezas de mayor importancia para el desarrollo profesional de los estudiantes, pues tan solo podremos mejorar nuestra 
práctica si somos conscientes de nuestros errores. De este modo, situamos al alumno en el centro del proceso de aprendizaje. Debemos aquí señalar que para que este recurso funcione con éxito, es necesario formar a nuestros alumnos, pues muchas veces una de las principales barreras que encontramos es que los estudiantes pueden sentirse reticentes a evaluarse a sí mismos, porque consideran que no cuentan con los conocimientos y habilidades necesarias o por falta de autoconfianza. Por lo tanto, será imprescindible que en clase potenciemos la inteligencia intrapersonal. De hecho la UNESCO en el artículo 9 de su Declaración mundial sobre la educación superior en el siglo XXI: visión y acción ya pone de manifiesto esta idea cuando dice "Las instituciones de educación superior deben formar a los estudiantes para que se conviertan en ciudadanos bien informados y profundamente motivados, provistos de un sentido crítico...". Por lo tanto, el objetivo de las instituciones de educación superior deberá ser formar a profesionales autónomos (entendida la autonomía como la capacidad para "asumir la responsabilidad del propio aprendizaje", Mora 2009: 182), reflexivos y metacognitivos, capaces de reflexionar sobre su propio proceso de aprendizaje, de autoevaluarse y, en definitiva, de controlar su propio aprendizaje. De esto modo, el profesor pasa a asumir el papel de guía y de apoyo en el proceso de aprendizaje, es decir, se convierte en un mero facilitador del conocimiento, mientras que el alumno asume la responsabilidad del proceso entendiendo que el aprendizaje ya no ocurre solamente dentro del aula, sino que debe producirse también fuera de ella.

Monereo i Font (1995: 78) indica que "promover la metacognición en el aula favorecerá el aprendizaje de los alumnos", idea con la que coincide Corsellis (2005), quien considera que el desarrollo de la autonomía en las aulas de interpretación resulta fundamental, pues se trata de una de las competencias básicas de todo intérprete profesional. Sin embargo, en el ámbito educativo, la capacidad de los estudiantes para controlar su propia actividad cognitiva no es suficiente, sino que entra en juego otra variable fundamental: la motivación (Pintrich \& De Groot 1990). García \& Doménech (1997) hablan de una motivación intrínseca, que procede del propio aprendiz y una motivación extrínseca, que procede de fuera y está más relacionada con otro tipo de satisfacciones que con la actividad en sí misma, por ejemplo, sacar buenas notas.

\subsection{La evaluación de la calidad en interpretación}

Tal y como indican Errico \& Morelli (2015), las primeras investigaciones sobre la calidad de la interpretación identificaban el contenido como criterio 
principal de valoración. Más adelante Collados (1998) demuestra empíricamente que otros aspectos como la monotonía de la voz influyen negativamente en la valoración global de una interpretación, incluso si la transmisión del sentido ha sido totalmente correcta.

Con el fin de garantizar la efectividad de la autoevaluación, hemos diseñado una rúbrica que sirva de guía a los alumnos. Para su elaboración se han tenido en cuenta cuáles son las competencias que debe desarrollar un alumno de Interpretación propuestas por Vanhecke \& Lobato (2009). De acuerdo con estas autoras, se requieren las siguientes aptitudes para ser un buen intérprete:

- Conocimiento de las lenguas de trabajo.

- Capacidad de análisis y de síntesis.

- Capacidad de extracción intuitiva del sentido del discurso.

- Capacidad de concentración.

- Buena memoria a corto y a medio plazo.

- Voz y presencia aceptables.

- Curiosidad intelectual, honradez intelectual y amplia cultura general.

- Tacto y sentido diplomático.

- Buena resistencia física y nerviosa y buena salud.

Como se puede observar, en esta lista de competencias se otorga una gran importancia al sistema paralingüístico que, de acuerdo con Cestero (2006: 60) está formado por "las cualidades y los modificadores fónicos, los indicadores sonoros de reacciones fisiológicas y emocionales, los elementos cuasi-léxicos y las pausas y silencios que a partir de su significado o de alguno de sus componentes inferenciales comunican o matizan el sentido de los enunciados verbales". Por lo tanto, además de reproducir el contenido del discurso adecuadamente, el intérprete deberá prestar atención a otros aspectos, como el tono, la intensidad de la voz o el ritmo que también influyen en la calidad de su trabajo.

Teniendo todos estos aspectos en cuenta se ha diseñado una rúbrica dividida en dos grandes bloques, por un lado, el análisis del contenido del discurso y, por otro lado, el análisis de la forma. De este modo, a la vez que llevan a cabo el visionado del vídeo, los alumnos pueden ir completando la plantilla. 


\begin{tabular}{|c|c|c|}
\hline CONTENIDO & Sí & No \\
\hline $\begin{array}{l}\text { 1. ¿Se aprecian en mi interpretación ideas sin coherencia debido a } \\
\text { una mala comprensión del original o falta de concentración y que } \\
\text { impiden seguir el discurso? }\end{array}$ & & \\
\hline $\begin{array}{l}\text { 2. ¿Hay omisiones en mi discurso meta? } \\
\text { En caso afirmativo, ¿qué tipo de omisiones? (cifras, nombres, } \\
\text { verbos, etc.) }\end{array}$ & & \\
\hline 3. ¿Termino todas las frases? & & \\
\hline 4. ¿Se aprecian en mi discurso contrasentidos? & & \\
\hline 1. ¿Se aprecian en mi discurso falsos sentidos? & & \\
\hline $\begin{array}{l}\text { 2. ¿Se aprecian en mi discurso calcos? } \\
\text { En caso afirmativo, ¿de qué tipo? }\end{array}$ & & \\
\hline $\begin{array}{l}\text { 3. ¿Se pone de manifiesto en mi discurso mi competencia resolutiva? } \\
\text { (ser capaz de aplicar otro tipo de estrategias cuando desconozco o } \\
\text { no recuerdo un término preciso? }\end{array}$ & & \\
\hline 4. ¿Soy capaz de autocorregirme? & & \\
\hline $\begin{array}{l}\text { 5. ¿En alguna parte del discurso me he beneficiado de mis } \\
\text { conocimientos previos/ cultura general? En caso afirmativo, } \\
\text { indica casos concretos: }\end{array}$ & & \\
\hline $\begin{array}{l}\text { 6. ¿Se aprecia en mi discurso un uso adecuado de la lengua meta } \\
\text { (expresiones idiomáticas, naturalidad, sintaxis correcta, ausencia } \\
\text { de literalidad, etc.)? }\end{array}$ & & \\
\hline $\begin{array}{l}\text { 7. ¿Logro transmitir toda la información o realizo una correcta } \\
\text { selección de las ideas más importantes? }\end{array}$ & & \\
\hline 8. ¿Utilizo en mi discurso un léxico amplio y variado? & & \\
\hline FORMA & & \\
\hline $\begin{array}{l}\text { 9. ¿Transmito inseguridad a la hora de formular mi discurso por un } \\
\text { excesivo uso de muletillas del tipo "eh..." o "mmm..."? }\end{array}$ & & \\
\hline $\begin{array}{l}\text { 10. ¿El ritmo de mi discurso es adecuado? (la velocidad permite } \\
\text { entender el discurso fácilmente y es consistente a lo largo de todo } \\
\text { el discurso). }\end{array}$ & & \\
\hline $\begin{array}{l}\text { 11. ¿La entonación es adecuada? (mi entonación contribuye a que el } \\
\text { público pueda comprender mejor el mensaje, p.ej., se enfatizan } \\
\text { partes clave del discurso). }\end{array}$ & & \\
\hline $\begin{array}{l}\text { 12. ¿La intensidad de mi voz es adecuada? ( } i e l \text { discurso se oye alto y } \\
\text { claro?). }\end{array}$ & & \\
\hline $\begin{array}{l}\text { 13. ¿Es mi dicción buena? (p.ej., no se aprecian problemas a la hora } \\
\text { de pronunciar ciertas consonantes). }\end{array}$ & & \\
\hline $\begin{array}{l}\text { 14. ¿Realizo un uso efectivo de estrategias estilísticas (cambio del } \\
\text { tono de voz, acentuación, velocidad del habla, énfasis, etc.)? }\end{array}$ & & \\
\hline 15. ¿Establezco contacto visual con el público a lo largo del discurso? & & \\
\hline 16. ¿Es mi postura corporal correcta? & & \\
\hline
\end{tabular}




\subsection{El diario de aprendizaje}

Como ya han puesto de manifiesto diversos investigadores (Doval 2005; Fernández Polo \& Cal Varela 2011; Jiménez 2011), el interés didáctico del diario de aprendizaje es que se trata de una herramienta que favorece la autonomía, pues es el alumnado el que asume la responsabilidad del proceso a través del cual adquirirá los conocimientos y las habilidades necesarias para mejorar su rendimiento profesional.

Nosotros proponemos que, una vez hecho el vídeo-análisis, los alumnos reflexionen por escrito sobre las fortalezas y debilidades de su interpretación. Se pretende que los estudiantes realicen este ejercicio semana tras semana, con la idea de que al verbalizar los aspectos que todavía tienen que mejorar, serán más conscientes de ellos y, casi de forma inconsciente, se centrarán en las sucesivas interpretaciones en mejorarlos.

\section{Conclusiones}

Las nuevas tecnologías y las exigencias del sistema de créditos ECTS obligan a los docentes a replantearse sus metodologías. En este contexto de cambio, resulta imprescindible que nos beneficiemos de las herramientas tecnológicas a nuestra disposición. En este artículo nos centramos en el trabajo autónomo del alumno y abogamos por el uso del vídeo análisis como vía de vinculación de la teoría y la práctica. Se trata de una herramienta muy valiosa en cuanto que sitúa al estudiante en el centro de su proceso de aprendizaje y un uso regular en la asignatura de Interpretación proporciona las siguientes ventajas:

- Desarrollar la capacidad analítica del alumnado, pues son capaces de detectar determinados parámetros y evaluarlos.

- Fomentar la metacognición de los alumnos como estrategia para mejorar su rendimiento.

- Impulsar su autonomía, ya que al colocarse en el centro del proceso de aprendizaje son ellos quienes lo controlan.

- Favorecer la motivación, porque, gracias al diario de aprendizaje pueden guardar un registro de todos los progresos. Cabe aquí destacar la importante labor del docente en este aspecto, pues a veces los alumnos tienden a centrarse solo en los aspectos negativos de su discurso, por lo que en esos casos es importante que el profesor/a intervenga para señalar las partes positivas.

Por lo tanto, consideramos que la combinación del vídeo análisis con el diario de aprendizaje contribuye a la mejora de la enseñanza de la interpretación, 
puesto que constituye un complemento efectivo al trabajo en el aula, que el alumno puede realizar de manera autónoma y que permite la integración de las nuevas tecnologías en la práctica docente.

\section{Referencias bibliográficas}

Alonso BaCigaluPe, Luis. (2009) El procesamiento de la información durante la interpretación simultánea: un modelo en tres niveles. Granada: Atrio.

Alonso BACIGAlUPE, Luis. (2005) "Pedagogía de la interpretación: investigación empírica sobre percepción de estudiantes." Sendebar 16, pp. 193-217.

Alonso BACIGALUPE, Luis. (2003) Investigación experimental en interpretación de linguas: primeiros pasos. Vigo: Universidade de Vigo.

Alonso BACIGALUPE, Luis. (2001) "La interpretación simultánea: una carrera de obstáculos." Sendebar 12, pp. 5-34.

AlONSO BACIGALUPE, Luis. (1999) "Metodología de iniciación a la interpretación simultánea." Perspectives: Studies in Translatology 7:2, pp. 253-263.

ARumí, Marta. (2009) "Nuevos retos en la formación de intérpretes: la integración del componente metacognitivo en el aula." Trans 13, pp. 149-162.

ARumí, Marta \& Laura DomíngueZ. (2013) "Sobre la necesidad de investigar la evaluación en el aula de interpretación." Cuadernos de ALDEEU 25, pp. 191-215.

BAER, Alejandro \& Bernt SCHNETTLER. (2014) "Hacia una metodología cualitativa audiovisual. El vídeo como instrumento de investigación social." En: Merlino, Aldo (ed.) 2014. Investigación cualitativa en Ciencias Sociales. Temas, problemas y aplicaciones. Buenos Aires: Cengage Learning, pp. 1-38.

BAIGORRI, Jesús. (2000) La interpretación de conferencias: el nacimiento de una profesión. Granada: Comares.

BAXTER, Robert Neal. (2012) "Modelo de formación básica en interpretación consecutiva dentro del marco de la reforma de Bolonia." Sendebar 23, pp. 61-77.

Bosch, Clara. (2012) Técnicas de interpretación consecutiva: la toma de notas. Manual para el estudiante. Granada: Comares.

Cestero, Ana Ma . (2006) "La comunicación no verbal y el estudio de su incidencia en fenómenos discursivos como la ironía." ELUA 20, pp. 57-77.

CORSELlis, Ann. (2005) "Training interpreters to work in the public services." En: Tennet, Martha (ed.) 2005. Training for the New Millennium: Pedagogies for translation and interpreting. Ámsterdam \& Filadelfia: John Benjamins, pp. 153-173.

De MANUel JeRez, Jesús. (2006) La incorporación de la realidad profesional a la formación de intérpretes de conferencias mediante las nuevas tecnologías y la investigación en la acción. Granada: Universidad de Granada. Tesis doctoral inédita. Versión electrónica: <https://hera.ugr.es/tesisugr/15891574.pdf> 
Domínguez, Lara. (2015) La evaluación para el aprendizaje de la interpretación de conferencias. Concepciones y prácticas de docentes y discentes en tres cursos de posgrado de Cataluña, Portugal y Canarias. Barcelona: Universitat Autònoma de Barcelona. Tesis doctoral inédita. Versión electrónica: <https://www.tdx.cat/ handle/10803/317965>

ERrico, Elena \& Mara Morelli. (2015) "La palabra a los oyentes: los comentarios del público en un cuestionario sobre la percepción de la calidad de la interpretación consecutiva de estudiantes en prácticas." MonTI Special Issue 2, pp. 281-301.

FAlCHIKOV, Nancy \& David Boud. (1989) "Student Self-Assessment in Higher Education: A Meta-Analysis." Review of Educational Research 59, pp. 395-430.

FERNÁNDEZ Polo, Francisco Javier \& Mario CAL VARELA. (2011) "Learning translation through the use of portfolios: description of an experience." Revista d'innovació educative 7, pp. 44-51

García, Francisco J. \& Fernando DOMÉNECH. (1997) "Motivación, aprendizaje y rendimiento escolar." Revista Electrónica de Motivación y Emoción 1:6, pp. 55-65.

García Doval, Fátima. (2005) "El papel de los portafolios electrónicos en la enseñanza-aprendizaje de las lenguas." Glosas didácticas: revista electrónica internacional de didáctica de las lenguas y sus culturas 14, pp. 112-119.

GILE, Daniel. (2009) Basic Concepts and Models for Interpreter and Translator Training. Ámsterdam: John Benjamins.

JuRAdo JimÉnEZ, Dolores. (2011) "El diario como un instrumento de autoformación e investigación." Qurriculum: Revista de teoría, investigación y práctica educativa 24, pp. 173-200.

KalinA, Sylvia. (2015) "Interpreter training and interpreting studies - Which is the chicken and which is the egg?" En: Dörte, Andres \& Martina Behr (eds.) 2015. To know how to suggest... Approaches to teaching conference interpreting. Berlín: Frank \& Timme, pp. 17-42.

LEDERER, Marianne. (1980) La traduction simultanée: fondements théoriques. Lille: Université de Lille.

LEDERER, Marianne. (1981) La traduction simultanée. Expérience et théorie. Paris: Lettres modernes.

MARTIN, Anne. (2015) "La formación en interpretación en España: pasado y presente." Monti Special Issue 2, pp. 87-110.

MONEREO I FONT, Carlos. (1995) "Enseñar a conciencia ¿Hacia una didáctica metacognitiva?" Aula de innovación educativa 34, pp. 74-80.

MORA, Miguel Ángel. (2009) "La autonomía del aprendizaje como marco pedagógico para el currículo de español como lengua extranjera." Marcoele. Revista de didáctica del español como lengua extranjera 9, pp. 181-194. 
Moro Cabero, Manuela \& Jesús Torres del Rey. (2008) La adaptación al espacio europeo de educación superior en la Facultad de Traducción y Documentación. Salamanca: Universidad de Salamanca.

PALOMARES, Ascensión. (2011) "El modelo docente universitario y el uso de nuevas metodologías en la enseñanza, aprendizaje y evaluación." Revista de educación 355, pp. 231-232.

Pintrich, Paul. R. \& Elisabeth V. De Groot. (1990) "Motivational and selfregulated learning components of classroom academic performance." Journal of Educational Psychology 82, pp. 33-40

RAMÓN, Elisa. (2010) La autonomía del alumno universitario de la especialidad de maestro en inglés: aplicación de un manual de autoestudio. Las Palmas: Universidad de Las Palmas de Gran Canaria. Tesis doctoral inédita. Versión electrónica: $<$ https://bibacceda01.ulpgc.es/bitstream/10553/5276/4/0627171_00000_0000. $\mathrm{pdf}>$

RODRíGUEZ, Raquel. (2006) "Diseño de entornos para el desarrollo de la autonomía en el aprendizaje." Aula abierta 86, pp. 89-104.

SANDRELli, Annalisa. (2015) "Becoming an interpreter: the role of computer technology." MonTI 2, pp. 118-138.

SELESKovitCH, Danica \& Marianne LederER. (1989) Pédagogie raisonnée de l'interprétation. Paris: Didier Érudition.

VANHECKE, Katrin \& Julia LOBATO PATRICIO. (2009) La enseñanza-aprendizaje de la interpretación consecutiva. Una propuesta didáctica. Granada: Comares.

Wiotte-FranZ, Claudia. (2001) Hermeneus und Interpres, zum Dolmetscherwesen in der Antike. Saarbrücken: Saarbrücker Druckerei und Verlag. 


\section{BIONOTA / BIONOTE}

LUCILA MARÍA PÉREZ FERNÁNDEZ es doctora en Traducción e Interpretación por la Universidad de Málaga. En su formación académica destacan los títulos de Licenciatura en Traducción e Interpretación (Universidad de Vigo), Licenciatura en Comunicación Audiovisual (Universidad de Salamanca), Título de Experto en Traducción en Contextos Especializados (Universidad de Córdoba), Máster en Diplomacia y Relaciones Internacionales (Escuela Diplomática de España), Posgrado en Enseñanza de lenguas (University of Bedfordshire) y Especialista Universitario en aprendizaje digital (CIESE-Comillas). Es Traductora Jurada nombrada por el MAEC. Su actividad investigadora se centra en los ámbitos de la traducción audiovisual, la traducción especializada y la enseñanza de lenguas. Actualmente trabaja como profesora en el departamento de Traducción e Interpretación de la Universidad Europea del Atlántico.

LuCila MARÍA PéREZ FernáNDEZ holds a PhD in Translation and Interpreting. She also holds a BA in Translation and Interpreting (University of Vigo), BA in Audiovisual Communication (University of Salamanca), Postgraduate certificate in Specialized Translation (University of Córdoba), MA in Diplomacy and International Relations (Spanish Diplomatic School), Postgraduate Certificate in Education (University of Bedfordshire), Postgraduate course in digital learning (CIESE-Comillas). She is a sworn translator named by the Spanish Ministry of Foreign Affairs. Her main research areas are audiovisual translation, specialized translation and language teaching. She currently teaches Translation courses at European University of the Atlantic. 
